\title{
Supervised and Unsupervised Classification of Cocoa Bean Origin and Processing using Liquid Chromatography-Mass Spectrometry
}

3

Santhust Kumar, ${ }^{1 \dagger *}$ Roy N. D’Souza, ${ }^{1 \dagger}$ Britta Behrends, ${ }^{1}$ Marcello Corno, ${ }^{2}$ Matthias S.

Ullrich, ${ }^{1}$ Nikolai Kuhnert ${ }^{1}$, and Marc-Thorsten Hütt ${ }^{1}$

${ }^{1}$ Department of Life Sciences and Chemistry, Jacobs University Bremen, Campus Ring 1, 28759 Bremen, Germany

$16 \dagger$ These authors contributed equally to this manuscript

$17 *$ Correspondence to:

18 Dr. Santhust Kumar (s.santhust@jacobs-university.de)

19 Department of Life Sciences \& Chemistry,

20 Jacobs University Bremen,

21 Campus Ring 1,

2228759 Bremen, Germany.

23 Phone: ++49 (421) 200-3549 


\section{ABSTRACT}

Liquid Chromatography-Mass Spectrometry (LC-MS) provides an unprecedented wealth of metabolomics information for food products, including insights into compositional changes during food processing. Here, we employed the largest available LC-MS dataset of around 300 cocoa bean samples to assess the capability of two popular multivariate classification methods, principal component analysis (PCA) and linear decomposition analysis (LDA), for studying bean geographic origin and responsible characteristic compounds.

The unsupervised method, PCA, only provides a limited separation in bean origin. Expectedly, the supervised method, LDA, provides a better origin clustering. However, it suffers from a strong, nonlinear dependence on the set of compounds used in the analysis. We show that for LDA a compound filtering criterion based on Gaussian intensity distributions dramatically enhances origin clustering of samples, thus increasing its predictive efficiency. In this form, the supervised method of LDA holds the possibility to identify potential markers of a specific origin.

Keywords: Theobroma cacao; LC-MS; principal component analysis (PCA); linear

\section{9 discriminant analysis (LDA); classification}

\section{1. Introduction}

41 The classification of cocoa based on quality has been a major challenge in the chocolate

42 industry, which is in industrial practice currently based on the rather simple cut-test, rather not

43 reflecting cocoa's vast chemical repertoire. Cocoa quality and geographic provenance define

44 cocoa prices and quality. Assurance of cocoa authenticity protects manufacturers and consumers alike from commercial fraud. 
46 Several of our research approaches have previously addressed classification of cocoa beans or

47 cocoa products based on variety of cocoa constituent classes (D'Souza et al., 2017; Kumari et al., 2018; Megías-Pérez et al., 2018; Sirbu et al., 2018) using information-rich techniques based on actual molecular fingerprints (Aculey et al., 2010; Magagna et al., 2017; Vázquez-Ovando et al., 2015), or low-resolution techniques based on sum parameters (Guehi et al., 2010).

The used methods have been shown to not only assess the degree of fermentation of a cocoa samples, but also to discriminate between its origin based on its metabolome fingerprint (Acierno et al., 2016, 2018; Bindereif et al., 2019; D’Souza et al., 2017; Kumari et al., 2018; Marseglia et al., 2016; Oliveira et al., 2016). Metabolic differences of the cocoa metabolome are a consequence of distinct agricultural practice, climate and soil influences (Adeniyi et al., 2019; Arévalo-Hernández et al., 2019; Asare et al., 2017; Ehiakpor et al., 2016; Kongor et al., $2016,2019)$ and are mainly based on genetic variability found in cocoa tree populations in different origin countries as illustrated by the group of Meinhardt on various occasions (Arevalo-Gardini et al., 2019; Gopaulchan et al., 2019; Lindo et al., 2018; Zhang and Motilal, 2016).

Liquid Chromatography-Mass Spectrometry (LC-MS) constitutes the most powerful technique for metabolomics analysis providing high resolution combined with high sensitivity. Cocoa products are among the most complex materials available to mankind with 10000 to 35000 individual peaks detectable in a single mass spectrum in processed cocoa (Kuhnert et al., 2013; Milev et al., 2014). When comparing a multitude of samples, powerful chemometric algorithms must be used to extract meaningful information from such datasets. At the same time, large sample numbers are challenging such algorithms to the extreme.

The state of the level of classification of origin of cocoa samples in the above-mentioned studies remained limited, because it is either at the level of continental regions or consider only 
70 few countries. Here we use a large data set comprising of 297 LC-MS profiles of aqueous methanolic extracts rich in polyphenolics and peptides (positive and negative ion modes) of unfermented or fermented cocoa beans, as well as selected cocoa liquors, sourced from various countries (10). The data has been gathered over a period of several years (2014-2018) and belongs to different stages of transformation in a typical cocoa processing pipeline. First, we demonstrate the limitations of the standard unsupervised go-to approach, principal component analysis (PCA) in clustering country of origin of cocoa samples. Second, we use a supervised approach, linear discriminant analysis (LDA), and show that its classification strongly depends on the number of features used in the analysis. We then outline a statistically motivated intuitive procedure for selecting features (compounds) and show that it greatly improves the outcome of LDA. We further show that this procedure of feature selection, which we refer to as Gaussian Feature Stability (GFS) requirement, also significantly improves prediction result for the country of origin achievable through LDA. The improvement in clustering of LDA can help in finding compounds which can differentiate between country of origin.

\section{Materials and Methods}

\subsection{Details of LC-MS data}

In the analysis we present here, a total of 297 LC-MS profiles of cocoa aqueous methanolic extracts rich in polyphenols and peptides was used. The LC-MS profiles are broadly categorized into two MS ion modes, three sample types and 10 origins (countries). These LCMS profiles have been obtained over a period of five years (2014-2018). The actual details of sample preparation, extraction, standard protocols, carrying out the LC-MS experiment and data collection are given in our earlier published reports (D'Souza et al., 2017, 2018). The 
93 number of LC-MS profiles with respect to ion modes, sample types and their country of origin

94 are provided in Table 1.

95 Positive and negative ion mode data jointly provide a more holistic approach enabling an

96 instant molecular snapshot of the coca bean chemical composition. Negative ion mode data

97 show a bias towards polyphenolic compounds, acids and carbohydrates, whereas positive ion

98 mode reveal a multitude of peptidic structures. Both modes are largely complementary with an

99 overlap of identified compounds of around 30-40\% depending on the sample type.

100

Table 1 Information about the LC-MS dataset. Country-wise and process-wise distribution

101 of number of LC-MS samples in the dataset used in this study.

\begin{tabular}{rcccc|cccc}
\hline & \multicolumn{3}{c}{ Positive ion mode } & \multicolumn{5}{c}{ Negative ion mode } \\
\cline { 2 - 9 } Unfermented & Fermented & Liquor & Sum & Unfermented & Fermented & Liquor & Sum \\
\cline { 2 - 9 } Cameroon & 4 & 4 & 0 & $\mathbf{8}$ & 4 & 4 & 0 & $\mathbf{8}$ \\
Ecuador & 8 & 3 & 6 & $\mathbf{1 2}$ & 3 & 3 & 7 & $\mathbf{1 3}$ \\
Ghana & 0 & 12 & 3 & $\mathbf{2 3}$ & 8 & 12 & 5 & $\mathbf{2 5}$ \\
Indonesia & 14 & 16 & 0 & $\mathbf{3 0}$ & 14 & 16 & 0 & $\mathbf{3 0}$ \\
Ivory Coast & 16 & 16 & 9 & $\mathbf{4 1}$ & 16 & 16 & 9 & $\mathbf{4 1}$ \\
Madagascar & 0 & 0 & 0 & $\mathbf{0}$ & 0 & 0 & 5 & $\mathbf{5}$ \\
Malaysia & 6 & 3 & 0 & $\mathbf{9}$ & 6 & 3 & 0 & $\mathbf{9}$ \\
San Thome & 0 & 0 & 0 & $\mathbf{0}$ & 0 & 0 & 5 & $\mathbf{5}$ \\
Tanzania & 3 & 9 & 0 & $\mathbf{1 2}$ & 3 & 9 & 4 & $\mathbf{1 6}$ \\
Sum & $\mathbf{5 4}$ & $\mathbf{6 3}$ & $\mathbf{2 3}$ & $\mathbf{1 4 0}$ & $\mathbf{5 4}$ & $\mathbf{6 3}$ & $\mathbf{4 0}$ & $\mathbf{1 5 7}$ \\
\hline
\end{tabular}

102

\subsection{Data pre-processing and cleaning}


104 We first processed the individual MS ion modes LC-MS data using MZMine (Pluskal et al., 2010). This yields a retention time aligned peak area list indicating the $\mathrm{m} / \mathrm{z}$ ratio, the retention time and the integrated peak areas of each sample. Then using a precompiled list of compounds

107 and their chemical formulas, corresponding $\mathrm{m} / \mathrm{z}$ values were de-convoluted and assigned for 108 determined in four ion types: [M-H], [M-2H], [M-3H], [2M-H], for negative ion mode, and $109[\mathrm{M}+\mathrm{H}],[\mathrm{M}+2 \mathrm{H}],[\mathrm{M}+3 \mathrm{H}],[2 \mathrm{M}+\mathrm{H}]$, for positive ion mode. Using this information, the 110 compounds detected in the LC-MS data were assigned to known structures, whenever possible

111 based on authentic references, tandem MS data or literature precedents, else the compound was 112 included, however, considered as 'Unknown_' suffixed with the $\mathrm{m} / \mathrm{z}$ value (e.g. 113 Unknown_865.1927). In cases where more than one compound matched an $\mathrm{m} / \mathrm{z}$ value, a 114 combined name, joined by 'or' was assigned. The assignment procedure was performed for 115 obtaining further insights, when possible, about the detected compounds. These processed data

116 were then combined with metadata about each sample in a single data structure, which 117 contained information about the sample type, origin and peak areas of various compounds. The 118 sum of peak area values belonging to each sample was normalized to 100 , so the peak area in

119 the sample represent relative percentage amount of compound in the LC-MS profile of the sample referred to the sum of all intensities (percentage normalized peak area). Henceforth, we refer to the percentage normalized peak area as the peak areas itself. Columns of compounds were sorted in descending order by their mean peak area across all samples, such that the compounds with higher mean peak areas are placed in earlier columns, and those with lesser

124 peak areas are placed in later columns.

\section{$125 \quad 2.3$ Unsupervised and supervised learning methods}

126 A couple of multivariate statistical analysis or 'machine learning' methods, both unsupervised

127 (e.g., PCA - Principal Component Analysis) and supervised (e.g., LDA - Linear Discriminant 
128 Analysis, Random Forests, Support Vector Machines, Neural Nets) have been used in this

129 study. These methods were applied using the popular scikit-learn module (Pedregosa et al.,

130 2011) of Python programming language. Standard scaling was implemented while performing

131 PCA. In case of Random Forests, Support Vector Machines, and Neural Nets, where it is

132 possible to vary algorithm specific parameters, default parameter settings in the

133 implementation in scikit-learn were used, to avoid additional complexity of dealing with

134 parameter specific algorithmic performance.

\subsection{Gaussian Feature Stability criterion}

136 The Gaussian Feature Stability (GFS) criterion is based on the test whether a set of values is

137 distributed normally, or not. If the values in the peak area list corresponding to a compound in

138 the LC-MS profiles, under a given sample type and belonging to a given origin, are found to

139 be normally distributed, we say that the compound satisfies the Gaussian Feature Stability

140 requirement. For testing normality of a set of values, we use the Shapiro-

141 Wilk (Shapiro and Wilk, 1965) test implemented in the popular Scipy module (Jones et al.,

142 2001) of Python programming language. We test normality at a p-value threshold of 0.05 .

\section{$143 \quad 2.5$ Null model}

144 First, we find out how many compounds (say $n$ ) satisfy GFS criterion under a group of samples.

145 Here, a group is comprised of samples belonging to a given country (say $X$ ), under a given

146 sample-type (say $Y$ ). Then we randomly choose $n$ compounds from the same group of origin

$147(X)$ and sample type $(Y)$. Using these $n$ randomly chosen compounds, we calculate prediction

148 from LDA. This procedure is repeated a number of times to obtain result statistics. The result

149 so obtained is referred to as result from null model.

\section{Results and Discussion}


151 For this study we have selected around 300 samples representing well the world of industrial

152 cocoa, including high production origins from Africa, South America and Asia over several

153 years of harvest. The dataset includes as well cocoa beans of different processing stages

154 reflecting chemical changes along the processing chain. In wine chemistry for example a

155 memory effect with respect to origin was recently proposed allowing improved origin

156 prediction following ageing (Roullier-Gall et al., 2014). In the main text we report results,

157 primarily, about the negative ion mode data. When appropriate or needed, corresponding plots,

158 results, etc., for the positive ion mode are reported in the Supporting Information.

\section{3.1. Classification of cocoa using PCA}

160 Principal component analysis (PCA), an unsupervised method of classification (James et al.,

161 2013), has been the first choice "go-to tool" in food chemistry analyses for exploring and

162 studying grouping relationships in samples (Aculey et al., 2010; Cordella, 2012; Granato et al.,

163 2018). However, PCA has been found of limited success in the study of country of origin of

164 cocoa beans (D’Souza et al., 2017; Kumari et al., 2018). In Figure 1, we show PCA score plots

165 for the negative ion mode data using unfermented, fermented and liquor samples of cocoa in

166 our analysis (using top 2000 compounds; see Supporting Information for positive mode data

167 and plots with different numbers of compounds). It can be seen that samples belonging to same

168 country (depicted by dots with same color in the PCA plots) tend to be present close by.

169 However, one can also clearly witness the mixing of samples belonging to different countries.

170 We note that this separation or mixing of samples further varies with (a) the number, and (b)

171 the actual set, of compounds used as features in the analysis. This points to a need for an

172 alternative approach for identifying marker-compounds, which can potentially allow

173 distinction between cocoa samples belonging to different country of origin. 

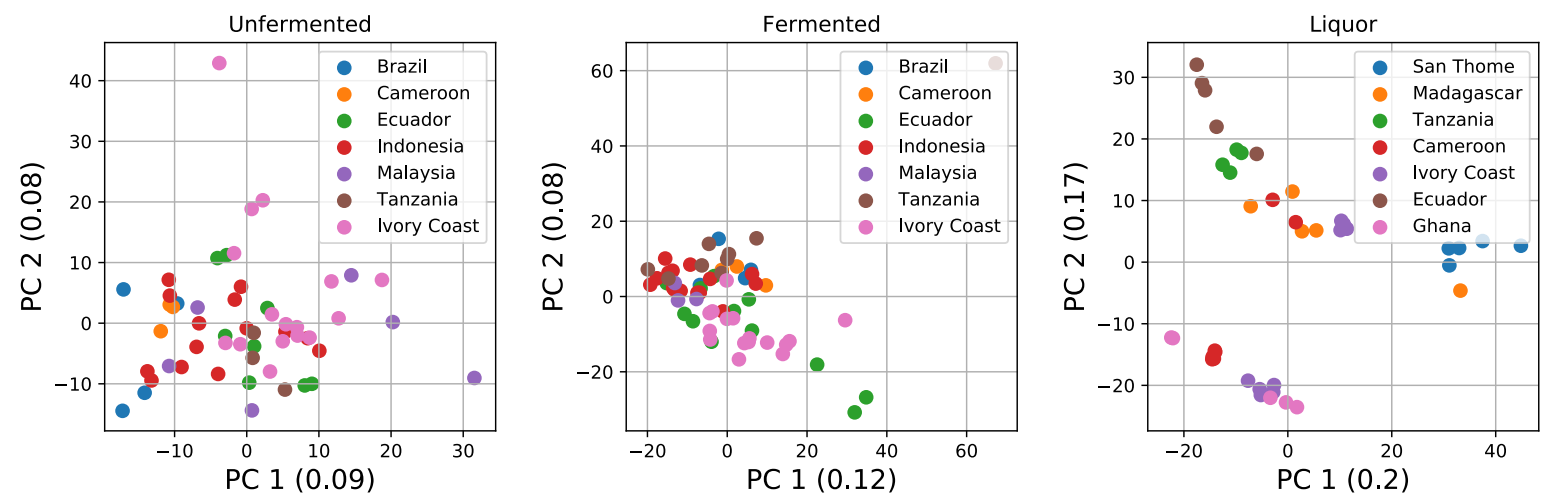
processing pipeline. The PCA gives a limited amount of grouping of samples belonging to same country.

179 We next applied linear discriminant analysis (LDA) (James et al., 2013) to the cocoa samples analyzed by LC-MS. LDA, as a supervised method of classification, uses the available class

181 information of the samples (in our case, country of origin) in order to find out axes, which give

182 best possible grouping of samples belonging to the same class (i.e., country, in our case). It achieves this by simultaneously minimizing the within-class variation and maximizing between-class variation. This is in contrast with PCA, which determines axes with most variation independent of the class the samples. In this way, LDA is better suited for determining compounds, which are good differentiators of samples belonging to different origins and compounds, which makes samples belonging to the same country similar.

In Figure 2, we show the LDA of unfermented, fermented, and liquor samples. For each sample type, the analysis is performed under four divisions, using: (a) all compounds, (b) the first 500 most abundant compounds, (c) the first 100 most abundant compounds, and (d) only the first

19110 most abundant compounds. It becomes apparent that the grouping of samples varies with 192 number of abundant compounds (or features) included prior to performing the LDA. A marked 
193 improvement in the grouping of samples belonging to same origin country is observed when

194 only including the top 30-50 most abundant compounds for the LDA. However, this soon

195 disappears as the number of compounds are further decreased. 
A
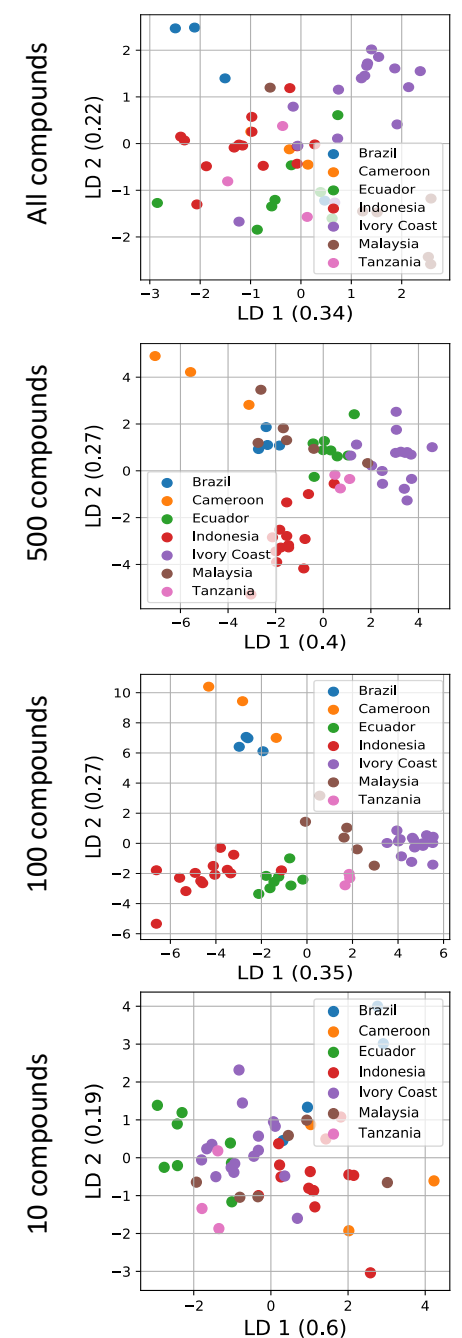

B
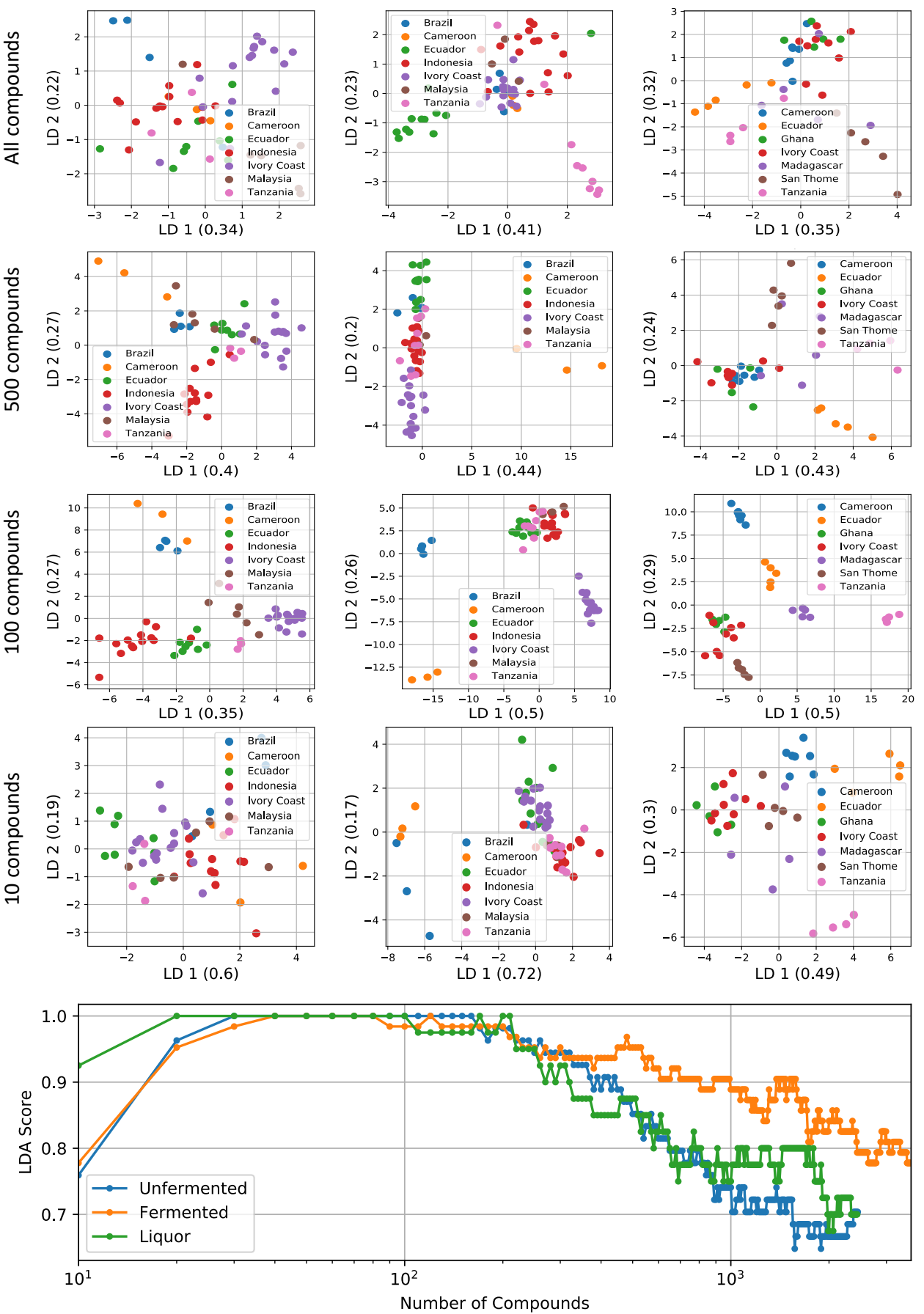

Figure 2 LDA for different sample-types using different number of compounds (features).

(A) The scatter plots show that the quality of grouping of samples depends on the set of compounds used in the analysis. (B) Non-linear variation of LDA-score (an estimate of clustering of same samples) as a function of number of compounds employed in the LDA. 
201 The quality of grouping of samples of same type can quantified by calculating the LDA score.

202 While in originality the LDA score is a measure of prediction power of the trained LDA model

203 upon the test dataset, it works well as a measure of clustering of the datapoints (or the

204 classifiability of the data), when the test dataset is kept the same as the training dataset. In

205 Figure 2B, we show the variation of LDA score with the number of compounds used for the

206 analysis. The classifiability from LDA changes with the number of compounds in a non-linear

207 manner. In the Supporting information, we provide results on positive ion mode and the

208 corresponding plots with the number of compounds displayed on a linear (not logarithmic)

209 scale. This points to the criticality of the number and set of compounds used as features in

210 performing the LDA, and how to select them.

\section{$211 \quad 3.3$ Filtering data for relevant features}

212 In general, feature selection is a factor that needs to be decided before performing a multivariate

213 analysis. Before performing LDA upon a given sample-type, we find out the set of compounds

214 which follow a Gaussian distribution under each country under the given sample type. We refer

215 to this filter as Gaussian Feature Stability (GFS) requirement (see section 2. Materials and

216 Methods). It is fair to expect that the distribution of intensities for a compound belonging to a

217 given country under a given process category is centered on some stable value, which might

218 be characteristic for this subset of samples. Otherwise, if the amount of the compound in the

219 samples belonging to a given country under a process category is not stable enough, it cannot

220 be considered as a reliable feature enabling classification for the samples. The reason for

221 instability can be varied: ranging from error in faithful detection of the compounds to the influence of other factors such as subsamples being procured in different batches over a period of time (as in our case the data is gathered over a period of few years). After obtaining such a list of compounds adhering to the GFS criterion, we use them as features for performing the 
225 LDA. Figure 3 shows the resulting improvement in the LDA brought about through this

226 approach. Essentially, the GFS criterion is able to reduce conflicting information from samples

227 belonging to same country. 


\section{Using all compounds}
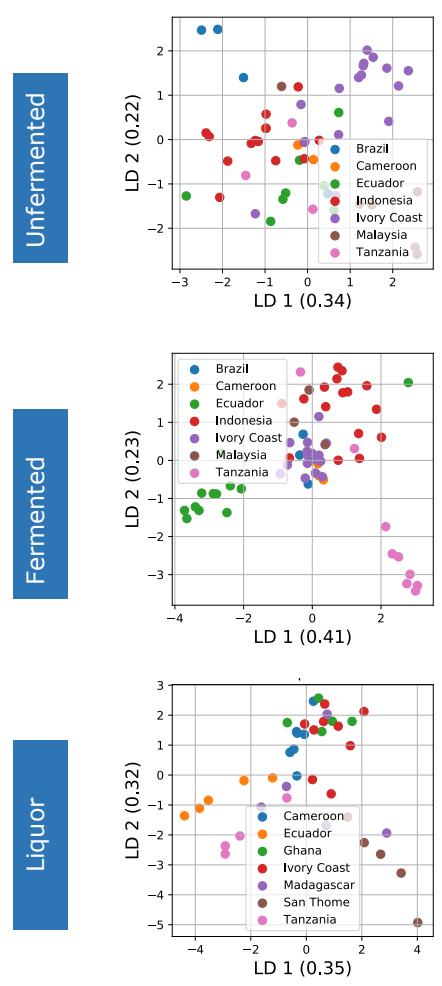

\section{After Gaussian feature stability requirement}
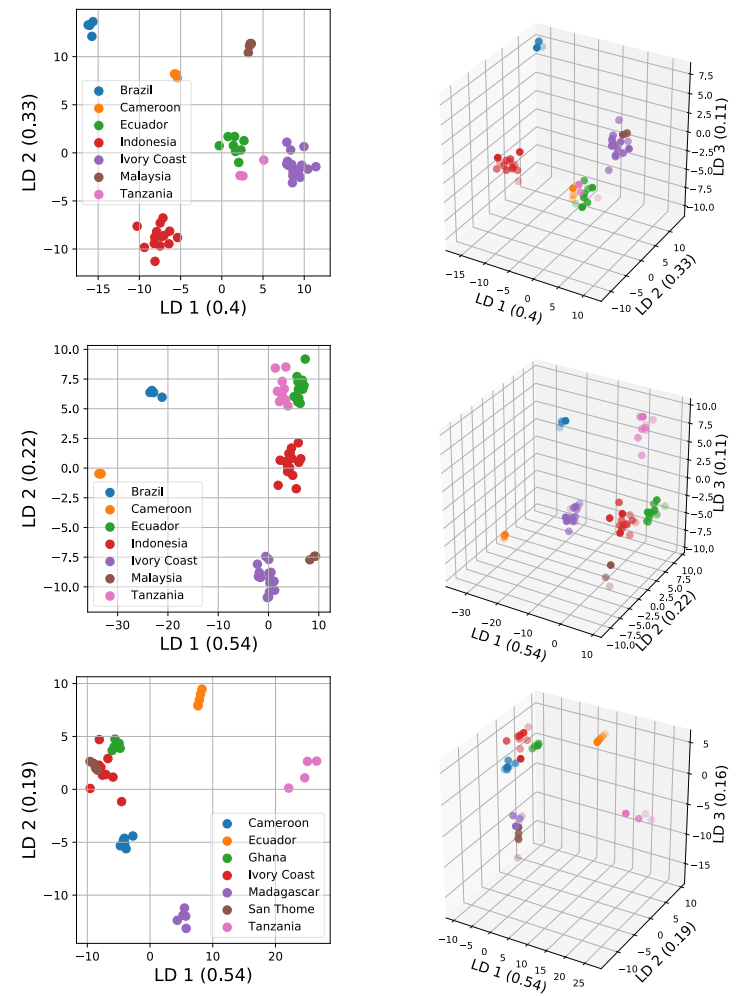

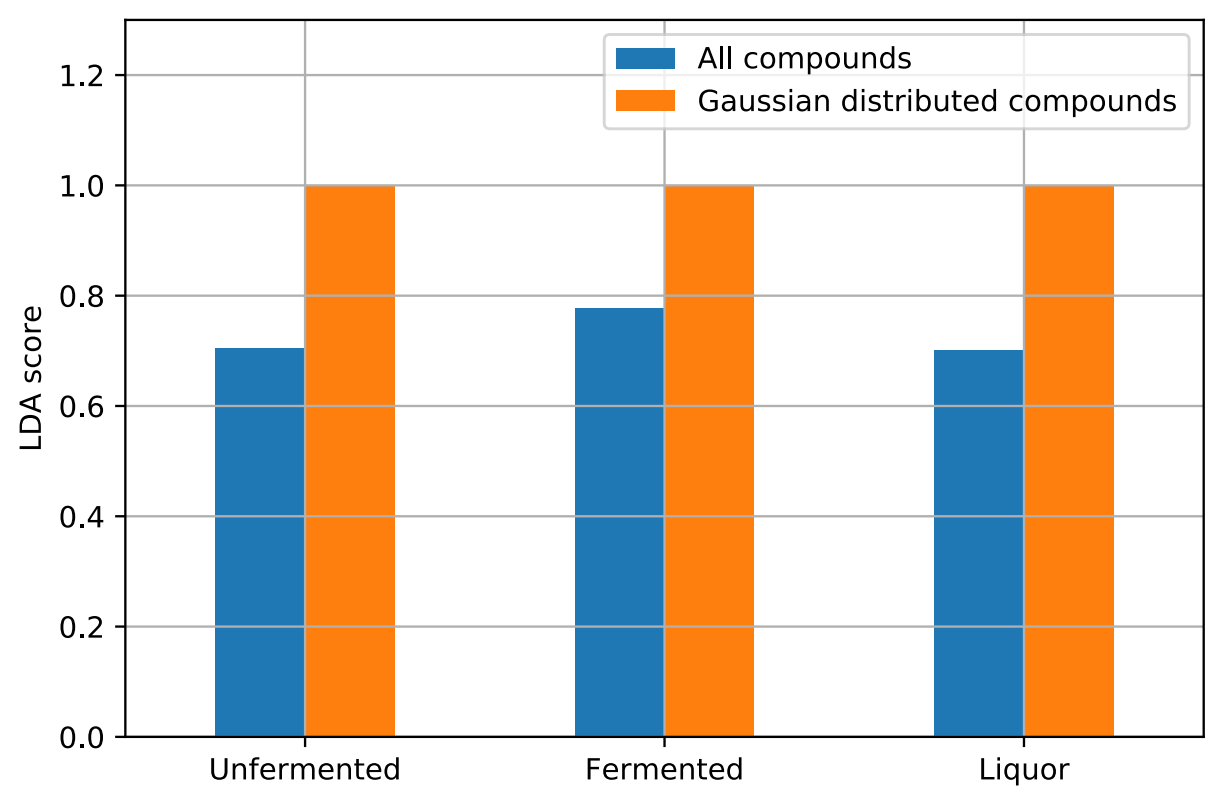

requirement. Scatter plots: Visual representation of clustering in LDA before and after application of GFS. Bar chart: Quantitative comparison of improvement in clustering in LDA after application of GFS. 
235 It should be pointed out that once the country clusters become clearly separated by the LDA,

236 the LDA score used above will no longer discriminate the amount of cluster separation (giving

237 a value close to 1 for all cases). The higher quality of the reduction of compounds based on

238 GFS will become clear in the following, when the LDA result is used for predictive purposes.

239 The origin-wise distributions of peak-list area of some metabolites and peptides obtained after

240 applying GFS requirement for unfermented, fermented, and liquors, are shown through

241 boxplots in Figure 4. The compounds have differing country-wise distribution, for e.g., $p$ -

242 Coumaroyl aspartate [M-H] serves as marker within the liquor category. Identifying

243 compounds with stable and differing distributions can be helpful in identification of origin

244 specific characteristic compounds. Figure 4 shows some selected examples of relative

245 quantities of marker compounds within the three stages of cocoa processing investigated. 

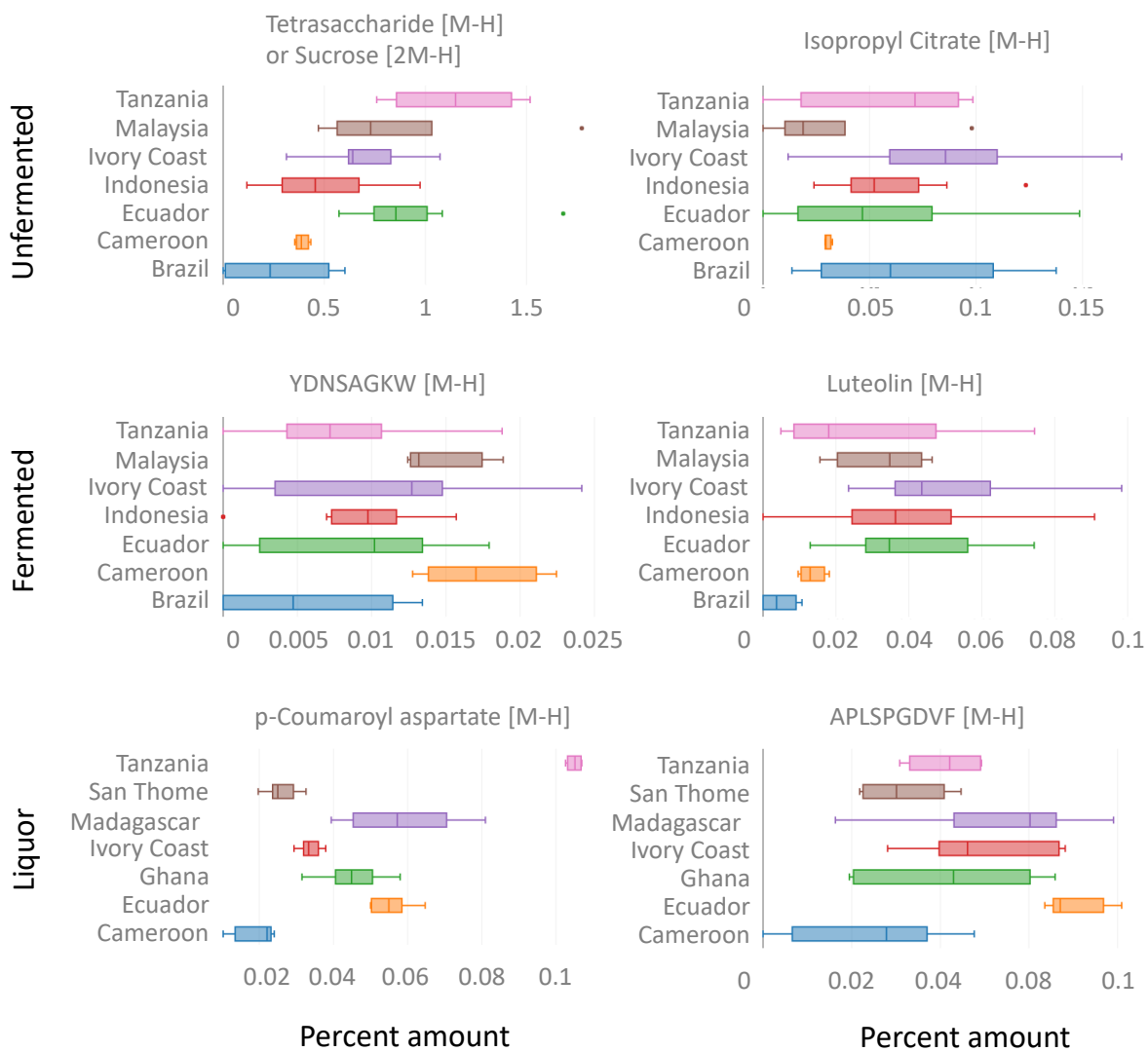

Figure 4 Profiles of compound concentration: The figure shows profiles of variation of concentration of compounds belonging to different countries for some compounds obtained after the application of Gaussian Feature Stability requirement. It can be seen that the countries have differing distributions of concentration of compounds.

\subsection{Effect of Gaussian Feature Stability on prediction through LDA}

252 Next, we study the effect of Gaussian Feature Stability (GFS) criterion on prediction of country

253 of origin through LDA. For each sample-type category, the list of compounds satisfying GFS

254 criterion was found. The sample dataset belonging to each category was divided into training 255 and test sets in ratio 3:1. After that, the LDA model was trained through the training dataset, and its predictive power was assessed through test dataset by calculating the LDA score. The whole procedure, from data splitting to prediction score, was repeated several times (100) to achieve result statistics. To put the outcome into perspective, a comparison was made with an 
appropriate null model. In this null model, the same number of compounds as obtained previously by GFS criterion, was randomly chosen from all compounds under a given category and used to obtain the prediction statistics (see 2. Materials and Methods). This procedure was repeated 100 times and was done for each category.

263 The result is plotted in Figure 5, with blue bars representing prediction power of LDA, when compounds satisfying GFS requirement were used, and orange bars represent the prediction power, when same number of randomly chosen compounds were used. It can be seen that higher predictive ability of LDA was achieved, when the compounds chosen through GFS criterion were used, as compared to the case when same number of randomly chosen compounds were used (2. Materials and Methods, Null model). Prediction using compounds satisfying GFS requirement through some popular machine learning algorithms is given in

Negative mode

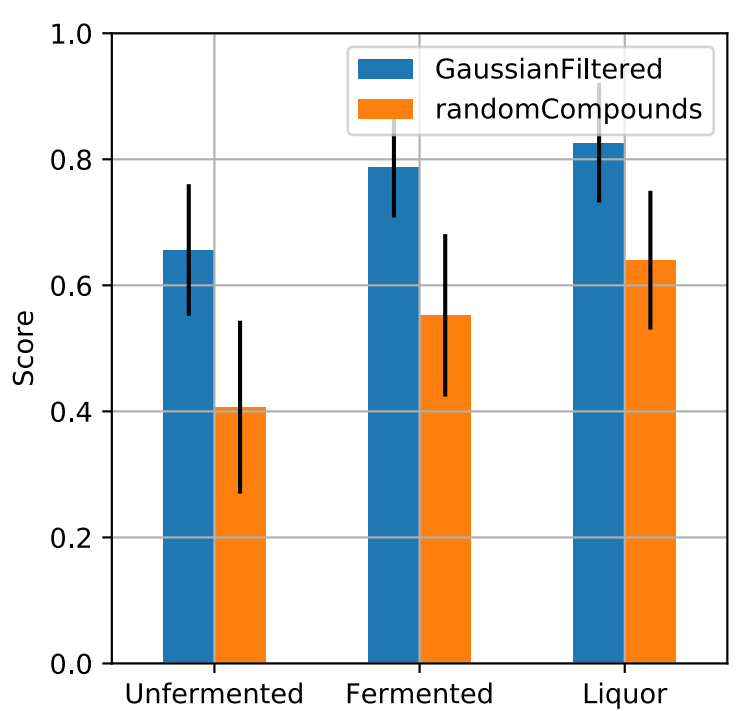

Positive mode

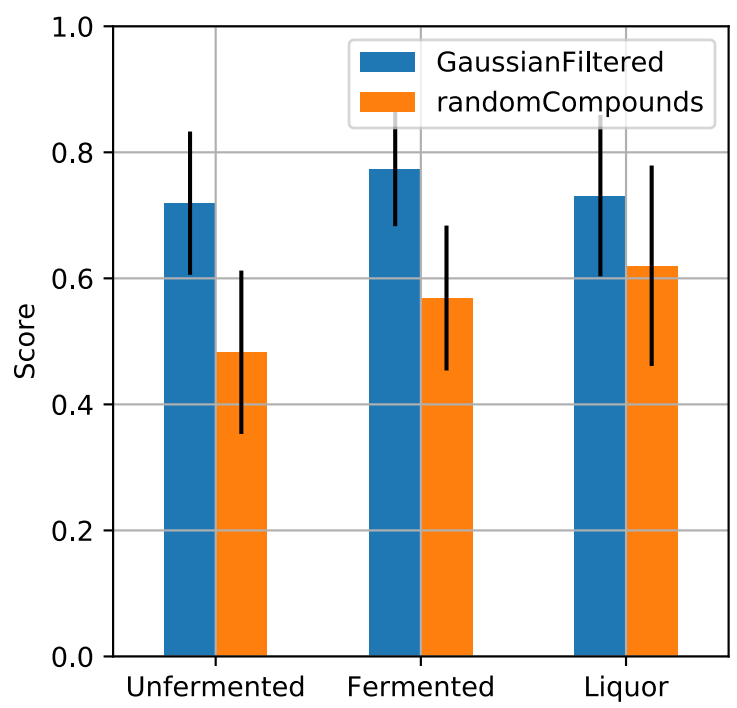




\section{Discussion and Conclusions}

280 We have used the biggest dataset of the LC-MS chemical profile of cocoa available to date to 281 study the feasibility of bean origin classification. Data from both positive and negative ion 282 modes have been analyzed.

283 PCA has often been used as a standard tool for studying such cases. PCA projects the samples

284 in the dataset into a lower dimensional space whose axes are formed by the principal components, which are vectors obtained from the linear combination of the original features, such that the variation is maximized along the principal components. The samples in the space thus obtained show most variation present in the dataset. The amount of contribution of the original feature (in our case, compound) in the determination of the principal axes can be read from a corresponding loading plot and is a measure of the importance of that feature (compound) in obtaining the represented variation in the dataset. If there appear groups of samples in the obtained space, then the compounds having high contribution in the

292 determination of principal axes are designated as compounds responsible for separating the

293 dataset into the obtained groups. There is quite some possibility that the compounds, which

294 have most variation, might be able to separate the dataset into some groups; however, it is not necessary. Only when the groups obtained after PCA show some correspondence to the original groups in the dataset, can one say with some confidence that compounds with relatively higher

297 weights in the principal axes of the space determined by PCA are responsible for the 298 differentiating one group from another. In summary, generally speaking, there is no binding 
reason for the compounds which show more variation in the dataset to also be the ones which

300 differentiate one group of samples from another in the dataset. While PCA has been shown to

301 successfully separate beans on basis of their fermentation status, it fails to separate beans on

302 the basis of their country of origin. Thus, there is a need to move beyond PCA to identify

303 compounds, which can differentiate beans of one country from another.

304 Since we have the country information about samples in our dataset, we employ LDA, which is a supervised learning method. Like PCA, LDA gives a lower dimensional space whose axes are represented by the obtained linear discriminants and in which samples in the dataset can be visualized. However, unlike PCA, LDA uses the class information about the samples in the data set to provide a space in which the within class variation is minimized and between class variation is maximized. In our case, this means that the samples belonging to the same country

310 should be as closely placed together as possible, and in turn these groups simultaneously should

311 be placed as far as possible. Again, like the PCA, the weighted contributions of the original

312 feature axes in the determination of linear discriminants determine the importance of features

313 in the observed separation, if any. Here we illustrate that LDA can be used to obtain a good

314 grouping of samples belonging to the same country, however, this depends on the subset and number of compounds used while performing LDA. The set of compounds is thus somewhat arbitrary. Further, a large number of low intensity compounds gets dropped out of analysis

317 some of which might still be relevant differentiators of origin of beans.

318 We coupled LDA with a statistical procedure of data cleaning which is intuitive and realistic:

319 We ask which of the compounds among the given set of compounds has a Gaussian distribution

320 (or nearly so) for each of the countries they are present in. One may argue that the characteristic features of a group with have their values distributed around some mean value along with some 
323 finds out statistically stable features, and on the other hand removes noise in the data. We then

324 perform LDA with the compounds, which pass this criterion that we call Gaussian Feature

325 Stability requirement. We notice a marked improvement in LDA classification of the samples

326 in the datasets.

327 Thus, this work makes an advancement in the direction of finding plausible differentiator

328 compounds for the origin of beans. Further, we couple a supervised learning method with a

329 novel, intuitive and realistically motivated criterion of feature selection and noise reduction.

\section{Declaration of competing interests}

331 The authors declare that they have no known competing financial interests or personal

332 relationships that could have appeared to influence the work reported in this paper.

\section{Acknowledgements}

334 We thank Nina Böttcher excellent technical support in sample logistics and preparation. This

335 work was funded by the COMETA project, which is financially supported by Barry Callebaut

336 AG. Barry Callebaut also provided samples for analysis. It has released the article for 337 publication.

\section{References}

340 Acierno, V., Yener, S., Alewijn, M., Biasioli, F., and van Ruth, S. (2016). Factors contributing to the

341 variation in the volatile composition of chocolate: Botanical and geographical origins of the cocoa

342 beans, and brand-related formulation and processing. Food Res. Int. 84, 86-95. 
343 Acierno, V., Alewijn, M., Zomer, P., and van Ruth, S.M. (2018). Making cocoa origin traceable:

344 Fingerprints of chocolates using Flow Infusion - Electro Spray Ionization - Mass Spectrometry. Food 345 Control 85, 245-252.

346 Aculey, P.C., Snitkjaer, P., Owusu, M., Bassompiere, M., Takrama, J., Nørgaard, L., Petersen, M.A., 347 and Nielsen, D.S. (2010). Ghanaian Cocoa Bean Fermentation Characterized by Spectroscopic and 348 Chromatographic Methods and Chemometrics. J. Food Sci. 75, S300-S307.

349 Adeniyi, S.A., de Clercq, W.P., and van Niekerk, A. (2019). Assessing the relationship between soil 350 quality parameters of Nigerian alfisols and cocoa yield. Agrofor. Syst. 93, 1235-1250.

351 Arevalo-Gardini, E., Meinhardt, L.W., Zuñiga, L.C., Arévalo-Gardni, J., Motilal, L., and Zhang, D. 352 (2019). Genetic identity and origin of "Piura Porcelana" - a fine-flavored traditional variety of cacao 353 (Theoborma cacao) from the Peruvian Amazon. Tree Genet. Genomes 15, 11.

354 Arévalo-Hernández, C.O., da Conceição Pinto, F., de Souza Júnior, J.O., de Queiroz Paiva, A., and 355 Baligar, V.C. (2019). Variability and correlation of physical attributes of soils cultivated with cacao 356 trees in two climate zones in Southern Bahia, Brazil. Agrofor. Syst. 93, 793-802.

357 Asare, R., Asare, R.A., Asante, W.A., Markussen, B., and Ræbild, A. (2017). INFLUENCES OF 358 SHADING AND FERTILIZATION ON ON-FARM YIELDS OF COCOA IN GHANA. Exp. Agric. $359 \quad 53,416-431$.

360 Bindereif, S.G., Brauer, F., Schubert, J.-M., Schwarzinger, S., and Gebauer, G. (2019). Complementary 361 use of 1H NMR and multi-element IRMS in association with chemometrics enables effective origin 362 analysis of cocoa beans (Theobroma cacao L.). Food Chem. 299, 125105.

363 Cordella, C.B.Y. (2012). PCA: The Basic Building Block of Chemometrics. Anal. Chem. 
364 D’Souza, R.N., Grimbs, S., Behrends, B., Bernaert, H., Ullrich, M.S., and Kuhnert, N. (2017). Origin-

365 based polyphenolic fingerprinting of Theobroma cacao in unfermented and fermented beans. Food Res.

366 Int. 99, 550-559.

367 D’Souza, R.N., Grimbs, A., Grimbs, S., Behrends, B., Corno, M., Ullrich, M.S., and Kuhnert, N. (2018).

368 Degradation of cocoa proteins into oligopeptides during spontaneous fermentation of cocoa beans. Food

369 Res. Int. 109, 506-516.

370 Ehiakpor, D.S., Danso-Abbeam, G., and Baah, J.E. (2016). Cocoa farmer's perception on climate

371 variability and its effects on adaptation strategies in the Suaman district of western region, Ghana.

372 Cogent Food Agric. 2, 1210557.

373 Gopaulchan, D., Motilal, L.A., Bekele, F.L., Clause, S., Ariko, J.O., Ejang, H.P., and Umaharan, P.

374 (2019). Morphological and genetic diversity of cacao (Theobroma cacao L.) in Uganda. Physiol. Mol.

375 Biol. Plants 25, 361-375.

376 Granato, D., Santos, J.S., Escher, G.B., Ferreira, B.L., and Maggio, R.M. (2018). Use of principal 377 component analysis (PCA) and hierarchical cluster analysis (HCA) for multivariate association between 378 bioactive compounds and functional properties in foods: A critical perspective. Trends Food Sci. 379 Technol. 72, 83-90.

380 Guehi, T.S., Zahouli, I.B., Ban-Koffi, L., Fae, M.A., and Nemlin, J.G. (2010). Performance of different 381 drying methods and their effects on the chemical quality attributes of raw cocoa material. Int. J. Food 382 Sci. Technol. 45, 1564-1571.

383 James, G., Witten, D., Hastie, T., and Tibshirani, R. (2013). Introduction to Statistical Learning.

384 Jones, E., Oliphant, T., Peterson, P., and others (2001). SciPy: Open source scientific tools for Python. 
385 Kongor, J.E., Hinneh, M., de Walle, D.V., Afoakwa, E.O., Boeckx, P., and Dewettinck, K. (2016).

386 Factors influencing quality variation in cocoa (Theobroma cacao) bean flavour profile - A review.

387 Food Res. Int. 82, 44-52.

388 Kongor, J.E., Boeckx, P., Vermeir, P., Van de Walle, D., Baert, G., Afoakwa, E.O., and Dewettinck,

389 K. (2019). Assessment of soil fertility and quality for improved cocoa production in six cocoa growing

390 regions in Ghana. Agrofor. Syst. 93, 1455-1467.

391 Kuhnert, N., Dairpoosh, F., Yassin, G., Golon, A., and Jaiswal, R. (2013). What is under the hump?

392 Mass spectrometry based analysis of complex mixtures in processed food - lessons from the

393 characterisation of black tea thearubigins, coffee melanoidines and caramel. Food Funct. 4, 1130-1147.

394 Kumari, N., Grimbs, A., D’Souza, R.N., Verma, S.K., Corno, M., Kuhnert, N., and Ullrich, M.S. (2018).

395 Origin and varietal based proteomic and peptidomic fingerprinting of Theobroma cacao in non-

396 fermented and fermented cocoa beans. Food Res. Int. 111, 137-147.

397 Lindo, A.A., Robinson, D.E., Tennant, P.F., Meinhardt, L.W., and Zhang, D. (2018). Molecular

398 Characterization of Cacao (Theobroma cacao) Germplasm from Jamaica Using Single Nucleotide

399 Polymorphism (SNP) Markers. Trop. Plant Biol. 11, 93-106.

400 Magagna, F., Guglielmetti, A., Liberto, E., Reichenbach, S.E., Allegrucci, E., Gobino, G., Bicchi, C., 401 and Cordero, C. (2017). Comprehensive Chemical Fingerprinting of High-Quality Cocoa at Early 402 Stages of Processing: Effectiveness of Combined Untargeted and Targeted Approaches for 403 Classification and Discrimination. J. Agric. Food Chem. 65, 6329-6341.

404 Marseglia, A., Acquotti, D., Consonni, R., Cagliani, L.R., Palla, G., and Caligiani, A. (2016). HR MAS

$4051 \mathrm{H}$ NMR and chemometrics as useful tool to assess the geographical origin of cocoa beans 406 Comparison with HR 1H NMR. Food Res. Int. 85, 273-281. 
407 Megías-Pérez, R., Grimbs, S., D’Souza, R.N., Bernaert, H., and Kuhnert, N. (2018). Profiling,

408 quantification and classification of cocoa beans based on chemometric analysis of carbohydrates using

409 hydrophilic interaction liquid chromatography coupled to mass spectrometry. Food Chem. 258, 284-

410294.

411 Milev, B.P., Patras, M.A., Dittmar, T., Vrancken, G., and Kuhnert, N. (2014). Fourier transform ion

412 cyclotron resonance mass spectrometrical analysis of raw fermented cocoa beans of Cameroon and

413 Ivory Coast origin. Food Res. Int. 64, 958-961.

414 Oliveira, L.F., Braga, S.C.G.N., Augusto, F., Hashimoto, J.C., Efraim, P., and Poppi, R.J. (2016).

415 Differentiation of cocoa nibs from distinct origins using comprehensive two-dimensional gas

416 chromatography and multivariate analysis. Food Res. Int. 90, 133-138.

417 Pedregosa, F., Varoquaux, G., Gramfort, A., Michel, V., Thirion, B., Grisel, O., Blondel, M.,

418 Prettenhofer, P., Weiss, R., Dubourg, V., et al. (2011). Scikit-learn: Machine Learning in Python. J.

419 Mach. Learn. Res. 12, 2825-2830.

420 Pluskal, T., Castillo, S., Villar-Briones, A., and Orešič, M. (2010). MZmine 2: Modular framework for 421 processing, visualizing, and analyzing mass spectrometry-based molecular profile data. BMC 422 Bioinformatics 11, 395.

423 Roullier-Gall, C., Boutegrabet, L., Gougeon, R.D., and Schmitt-Kopplin, P. (2014). A grape and wine 424 chemodiversity comparison of different appellations in Burgundy: Vintage vs terroir effects. Food 425 Chem. 152, 100-107.

426 Shapiro, S.S., and Wilk, M.B. (1965). An Analysis of Variance Test for Normality (Complete Samples).

427 Biometrika 52, 591-611. 
428 Sirbu, D., Grimbs, A., Corno, M., Ullrich, M.S., and Kuhnert, N. (2018). Variation of triacylglycerol

429 profiles in unfermented and dried fermented cocoa beans of different origins. Food Res. Int. 111, 361-

$430 \quad 370$.

431 Vázquez-Ovando, A., Molina-Freaner, F., Nuñez-Farfán, J., Betancur-Ancona, D., and Salvador-

432 Figueroa, M. (2015). Classification of cacao beans (Theobroma cacao L.) of southern Mexico based on

433 chemometric analysis with multivariate approach. Eur. Food Res. Technol. 240, 1117-1128.

434 Zhang, D., and Motilal, L. (2016). Origin, Dispersal, and Current Global Distribution of Cacao Genetic

435 Diversity. In Cacao Diseases: A History of Old Enemies and New Encounters, B.A. Bailey, and L.W.

436 Meinhardt, eds. (Cham: Springer International Publishing), pp. 3-31.

437

438 